\title{
Carbon Nanotubes: Release to the Environment, Toxicity and Control Technologies
}

\author{
Jing Wang \\ Institute of Environmental Engineering, ETH Zurich, \\ Rämistrasse 1018092 Zurich, Switzerland \\ jing.wang@ifu.baug.ethz.ch
}

Carbon nanotubes (CNTs) represent a type of fascinating nanomaterial which gained numerous applications due to their special mechanical, electrical, thermal and optical properties. On the other hand, CNTs are one of the most heavily studied nanomaterials for their potential impacts on human and environment. Toxicity studies using CNTs reveal important mechanisms for their biological effects, however, CNTs are seldom used alone as a consumer product; instead they are often applied as additives in composites. Therefore, the release of CNTs from composites into the environment and the toxicity of the CNTs mixed with other released particles are essential for the CNT risk assessment. We showed for the first time release of free-standing individual CNTs from nanocomposites during abrasion processes, developed a new quantification method to measure the exposed CNTs in the abraded particles, and investigated their toxicity. The results generally demonstrated that the released CNTs under the investigated situations pose no substantial acute risk for workers and consumers. The CNTs tend to agglomerate due to their high aspect ratios and van der Walls forces in air and water. We used the concept of fractal geometry to develop a model for CNT agglomerates, which enables calculation of the agglomerate properties such as the mass, porosity, effective density, surface area and characteristic diameters. Filtration is an effective technology for CNT control and results using several types of filters will be shown. 